\title{
MÉTABOLISME DE LA NICARBAZINE CHEZ LE POULET
}

PAR

\author{
A. C. FRANGois et A. J. PIHET
}

Service de Biochimie et de Nutrition, C. N. R. Z., Jouy-en-Josas (S.-et-O.).

\section{SOMMAIRE}

La nicarbazine, administrée à des volailles pendant huit semaines, aux taux de 0,0125 p. Ioo et $0,02 \mathrm{p}$. I Oo, provoque dans le muscle et dans le foie une accumulation de la fraction dinitrocarbanilide. Les taux retrouvés sont respectivement, dans le muscle de 0,00012 et de $0,00016 \mathrm{p}$. I00 pour les deux doses et dans le foie de 0,0003 et 0,0007 p. Ioo de DNC. La fraction hydroxydiméthylpyrimidine (HDP) n'a été retrouvée ni dans le muscle, ni dans le foie.

La nicarbazine est un complexe moléculaire de 4-4' dinitrocarbanilide (DNC) et de 2-hydroxy 4-6 diméthylpyrimidine (HDP). Elle jouit de propriétés coccidiostatiques intéressantes pour l'élevage avicole (CuCkLER et al, I955); à cet effet on l'incorpore dans les aliments à la. dose de o,or 25 p.IOo. Toutefois, elle entre dans la catégorie des substances auxiliaires de l'alimentation, c'est-à-dire de substances qui ne possèdent pas de propriétés nutritionnelles intrinsèques. Le problème des résidus qu'elle est susceptible de laisser dans les tissus des animaux traités présente donc un grand intérêt pour l'hygiéniste.

\section{MATÉRIEL ETT MÉTHODES}

a) Animaux et régime. - Deux lots d'animaux ont été constitués. L'un de ces lots recevait dans sa ration 0,0125 p. Ioo de nicarbazine, c'est-à-dire la dose utilisée dans la pratique.Dans le dessein d'étudier l'influence de taux plus élevés, un autre lot recevait $0,02 \mathrm{p}$. Ioo de cet anticoccidien.

Après huit semaines, les animaux étaient sacrifiés et l'on prélevait 
des échantillons de tissu musculaire (muscle pectoral, nuscles de la cuisse) ainsi que le foie. Chacun des lots comprenait dix animaux; pour les dosages, les tissus et crganes homologues de deux animaux étaient réunis. La recherche des fractions dinitrocarbanilide et hydroxydiméthylpyrimidine était également effectuée chez des animaux témoins ne recevant pas de nicarbazine.

b) Méthode de dosage. - Deux méthodes différentes ont été adoptées, l'une permettant de doser la fraction DNC et, l'autre, la fraction HDP, afin d'étudier le nétabolisme de ces deux fractions de la nicarbazine. $I_{i} a$ première de ces méthodes était une adaptation de la technique proposée par Polis et al (I956) et la seconde d'entre elles constituait une adapta. tion de la méthode de Szalkowski (I957).

\section{RÉSULTATS}

Afin d'étudier la validité de ces différentes méthodes dans le cas de leur application aux organes et tissus de poulets, nous avons procédé à des surcharges de quantités connules de nicarbazine. Les résultats obtenus montrent que les récupérations basées sur le dosage de la fraction DNC, ou de la fraction HIP, sont de l'ordre de go p. Ioo pour des taux de nicarbazine variant rle 0,00025 à $0,0005 \mathrm{p}$. roo. La réaction qui porte sur la fraction DNC est extrêmement sensible et permet le dosage de o,ooor p. xoo de cette substance. Nous avons vérifié que la réaction était totalement négative avec les muscles et le foie des animaux témoins.

I'application de ces deux méthodes aux tissus et organes obtenus à partir des animaux auxquels nous avons administré respectivement 0,0125 et $0,0200 \mathrm{p}$. Ioo de nicarbazine dans l'aliment, a donné les résultats résumiés dans les tableaux I et II.

\section{TABLEAT I}

Fractions DNC et HDP retronvées dans le muscle et dans le foie de poulets recevant 0,0125 p. 100 de nicarbazine dans leur ration (p. ,oo de tissu frais).

\begin{tabular}{|c|c|c|c|c|}
\hline \multirow[b]{2}{*}{ Animaux } & \multicolumn{2}{|c|}{ Nuscle } & \multicolumn{2}{|c|}{ loie } \\
\hline & $\mathrm{DNC}$ & HDP & $\mathrm{DNC}$ & IIDP' \\
\hline$I$ & 0,0001 & néant & 0,00035 & néant \\
\hline $2 \ldots \ldots \ldots$ & 0,0001 & - & 0,00035 & -- \\
\hline $3 \ldots \ldots$ & 0,0002 & - & $0,000+$ & - \\
\hline $4 \ldots \ldots$ & 0,0001 & - & 0,0002 & - \\
\hline $5 \ldots \ldots \ldots$ & 0,0001 & - & 0,0002 & - \\
\hline Moyenne... & 0,00012 & & 0,0003 & \\
\hline
\end{tabular}

I1 n'a pas été possible de retrouver la moindre trace d'hydroxydiméthylpyrimidine ni dans le muscle ni dans le foie. Toutefois, ceci n'est 
vraisemblablement pas dû à une insuffisance de la sensibilité de la méthode, car nous avons vérifié, au moyen de surcharges, qu'un taux de 0,00025 p. IOo de HDP était dosable dans les tissus.

TABLEAU II

Fractions DNC et HDP retrouvées dans le muscle et dans le foie de poulets recevant $0,020 \mathrm{p}$. IoO de nicarbazine dans leur ration.

\begin{tabular}{|c|c|c|c|c|}
\hline \multirow[b]{2}{*}{ Animaux } & \multicolumn{2}{|c|}{ Muscle } & \multicolumn{2}{|c|}{ Foie } \\
\hline & DNC & $\mathrm{HDI}^{\prime}$ & DNC & IIDP \\
\hline$\overline{6} \ldots \ldots$ & $\overline{0,0002}$ & néant & $0, \overline{0007}$ & néant \\
\hline $7 \ldots \ldots$ & 0,00015 & $\ldots$ & 0,0006 & - \\
\hline $8 \ldots \ldots$ & 0,00015 & - & 0,0009 & - \\
\hline $9 \ldots \ldots$ & 0,00015 & - & 0,0007 & - \\
\hline ro..... & 0,00015 & - & 0,0007 & - \\
\hline Moyenne... & 0,00016 & & 0,0007 & \\
\hline
\end{tabular}

\section{DISCUSSION}

I,es résultats précédents montrent que l'administration orale de nicarbazine provoque un passage de cette substance dans les tissus $\mathrm{du}$ poulet. Toutefois, ce stockage porte essentiellement sur la fraction DNC et le taux retrouvé dans le muscle est faible : de l'ordre de o,ooor2 p. Ioo poir un régime contenant o,oI25 p. Ioo de nicarbazine. Ce taux est plus élevé dans le foie : 0,0003 p. Ioo pour les doses alimentaires usuelles de nicarbazine, et 0,0007 p.Ioo pour un régime contenant 0,02 p. Ioo de cet anticoccidien. I a quantité retrouvée dans le tissu musculaire correspond donc approximativement à I mg de DNC par $\mathrm{kg}$. En conséquence le consommateur de poulet ingérerait de l'ordre de $0,2 \mathrm{mg}$ de cette substance au cours d'un repas, soit environ 3 microgrammes par $\mathrm{kg}$ corporel.

Ces résultats sont sur certains points en accord avec ceux de PORTER et GILFILLAN (I955) pour ce qui concerne le métabolisme des fractions DNC et HDP : chez des poulets recevant pendant douze semaines un régime contenant o,or p. Ioo de nicarbazine, ces auteurs n'observaient aucun résidu de HDP dans le muscle et le foie, et retrouvaient 5,3 micromolécules de DNC par ml de plasma. Toutefois, alors que PORTER et GILFILLAN ne retrouvaient pas de DNC dans le muscle, chez des oiseaux recevant 0,0 I p. Ioo de nicarbazine dans leur ration, les résultats obtenus dans la présente expérience permettent de mettre en évidence $\mathrm{I}, 2 \mathrm{mg}$ de DNC par kg de tissu musculaire chez des animaux qui reçoivent un régime à 0,0125 p. I0o. Dans le cas des animaux qui reçoivent 0,02 p. Ioo de nicarbazine, les résultats obtenus par PorTer et Gilfirlan et par nous-mêmes sont du même ordre de grandeur ( $\mathrm{r}, 8$ contre $\mathrm{r}, 6 \mathrm{mg})$. 
Ėn revanche, nous n'avons retrouvé de résidu de HDP ni dans le muscle, ni dans le foie, alors que PORTER et GILFIILAN retrouvaient cette fraction dans le tissu hépatique. Fn utilisant de la nicarbazine marquée, Clark et al. (I956) ont également montré que seule la fraction DNC s'accumule dans le muscle de poulet. On sait que la dinitrocarbanilide s'élimine rapidement des tissus lorsqu' on cesse la distribution alimentaire de nicarbazine (PorTER et Gilfililan, I955; Clark et a1., I956). Néanmoins, les problèmes relatifs à la toxicologie de cette substance mériteraient d'être étudiés.

\section{SUMMARY}

The metabolism of nicarbazin, a complex composed of one molecule of 2-hydroxy-4-6-dimethylpyrimidine (HDP) and of one molecule of 4-4' dinitrocarbanilide (DNC), was studied in the chicken. Three groups of ten animals were established : a control group, a group receiving o.0I25 p. Ioo and another group receiving $0.02 \mathrm{p}$. Ioo nicarbazin in the food. The animals were slaughtered after eight weeks on this diet, and the hydroxymethylpyrimidine and dinitrocarbanilide content of the muscle and liver was studied: by the colorimetric methods of PoLIN et al. (I956) and Szal.Kowski (I957).

No HDP residue was detectable either in the muscle or in the liver. On the other hand 0.00012 and $0.00016 \mathrm{p}$. 100 of $\mathrm{DNC}$ were found in the muscle of the groups receiving 0.0125 and $0.02 \mathrm{p}$. I00 nicarbazin, respectively. The corresponding liver contents amounted to 0.0003 and $0.0007 \mathrm{p}$. I00.

\section{REFFÉRENCES BIBLIOGRAPHIQUES}

Clark I., Geoffroy R. F., Gilfillan J. L. et Porter C. C., I956. Absorption and elimination of carbon I4 labelled nicarbazin by chickens. Proc. Soc. Exp. Biol. Med., 4-6, 9I.

Cuckler A. C., x955. The antiparasitic activity of nicarbazin. Poult. Sci., 34, 98.

Polin D., Gilfillian J. F., O'TT W. H. et PORTER C. C., I956. 4-4' Dinitrocarbanilide in egg yolks from hens fed nicarbazin. Poult. Sci., 35, I367.

Porter C. C. et Gilfillan J. F., I955. The absorption and excretion of orally administrated nicarbazin by chickens. Poult. Sci., 34, 995.

Szalkowski C. R., 1957. Report on nicarbazin in feeds. Ass. off. agric. Chem., 40, 469 . 